\title{
Wie können Traditionelle Medizin sowie Komplementäre und Alternative Medizin durch die Weltgesundheitsorganisation konkret unterstützt werden?
}

\author{
Stefan Mumenthaler \\ Thun, Schweiz
}

\section{Schlüsselwörter}

Weltgesundheitsorganisation, WHO - Traditionelle Medizin .

Komplementäre und Alternative Medizin, CAM - Gesundheitssystem . Beijing-Deklaration

\section{Zusammenfassung}

Die Weltgesundheitsorganisation (WHO) engagiert sich auf verschiedenen Ebenen für die Gesundheit der Menschen dieser Welt. Über 210 verschiedene Programme und Projekte listet die internationale Organisation über ihre Tätigkeiten auf. Die Traditionelle Medizin (TM) und die Komplementäre und Alternative Medizin (CAM) decken weltweit gesehen einen grossen Teil der medizinischen Grundversorgung ab. So stellt sich hier die Frage, ob und wie die TM/CAM durch die WHO mit ihrer Arbeit auf globaler Ebene und in der europäischen Region unterstützt werden kann. Dabei findet sich ein Engagement für Themen der TM/CAM seit über 40 Jahren - mit beachtenswerten Resolutionen und Deklarationen dazu. Die Entwicklung in den letzten 10 Jahren und der erste «WHO-Kongress für Traditionelle Medizin» in Peking, China, im Jahr 2008 zeugen von einem Engagement zugunsten von TM/ CAM: Die WHO fordert ihre 193 Mitgliedsstaaten auf, im Rahmen ihrer Möglichkeiten Schritte zu unternehmen, um die TM/CAM in die nationalen Gesundheitssysteme zu integrieren. Sie bietet dabei interessierten Staaten ihre Unterstützung an. Drei von sechs WHO-Regionalbüros führen bereits eigene Programme für die TM. Das Regionalbüro für Europa führt hingegen kein eigenes Programm für TM/CAM. Unter dem Gesichtspunkt der angestrebten intensiveren Zusammenarbeit der WHO mit der Europäischen Union wird der Frage nachgegangen, wie das Regionalbüro die auf globaler Ebene gefassten Resolutionen zu TM/ CAM in Europa umsetzen will. Eine Unterstützung des Regionalbüros durch die internationale Ebene der WHO sowie durch regionale Stakeholder und Interessengemeinschaften der CAM wird zur Diskussion gestellt.

\author{
Keywords \\ World Health Organization, WHO - Traditional Medicine . \\ Complementary and Alternative Medicine, CAM · Health system . \\ Beijing Declaration
}

\section{Summary}

How Can Traditional Medicine and Complementary und Alternative Medicine Be Concretely Supported by the World Health Organization? The World Health Organization (WHO) is dedicated to promoting the health of all people. Its activities are demonstrated by more than 210 different programs and projects operating at varying levels. Traditional Medicine (TM) and Complementary and Alternative Medicine (CAM) account for a large part of primary health care worldwide. One issue to consider is if and how the WHO can support TM/CAM at a global level and at the level of the European region. The organization's commitment to the topics of holistic medicine for over 40 years as well as its significant resolutions and declarations regarding TM/CAM must be noted. The developments in the last 10 years, combined with the first 'WHO Congress for Traditional Medicine' held in Beijing, China, in 2008, are further examples of the organization's commitment to TM/ CAM. The WHO is also advocating for a shift towards integrating TM/ CAM within the national health systems of all 193 member states to the best of their abilities. In order to realize this aim, the WHO is extending assistance to interested member states. Three of the six WHO Regional Offices are already operating their own programs for TM/CAM. The Regional Office for Europe, however, does not have its own TM/CAM program. With respect to the desired intensified cooperation between the WHO and the European Union, the following question arises: How does the Regional Office intend to implement the globally conceived TM/CAM resolutions in Europe? TM/CAM support for the Regional Offices by the WHO at the international level, in conjunction with the support by stakeholders and interest groups at the regional level, is discussed at length in this paper.

\section{KARGER \\ Fax +497614520714 Information@Karger.de} www.karger.com
Stefan Mumenthaler

Bälliz 42, 3600 Thun, Schweiz

Tel. +41332227773

info@activita.com 


\section{Mots-clés}

Organisation mondiale de la santé, OMS - Médecine traditionnelle . Médecines complémentaires et alternatives, MCA .

Système de santé · Déclaration de Pékin

\section{Résumé}

Comment l'Organisation mondiale de la santé peut-elle concrètement soutenir la médecine traditionnelle ainsi que les médecines complémentaires et alternatives?

L'Organisation mondiale de la santé (OMS) s'engage à différents niveaux en faveur de la santé des êtres humains dans le monde. À travers ses activités, cette organisation internationale compte plus de 210 programmes et projets différents. Du point de vue mondial, la médecine traditionnelle (MT) et les médecines complémentaires et alternatives (MCA) couvrent une grande partie des soins médicaux de base. La question se pose donc de savoir si et comment l'OMS peut soutenir la MT et les MCA par son travail à un niveau global et en Europe. Il existe pourtant, depuis plus de 40 ans, un engagement en faveur de thèmes liés à la MT et aux MCA, avec des résolutions et déclarations notables à ce sujet. Le développement au cours de ces 10 dernières années et le premier «congrès de l'OMS sur la médecine traditionnelle» à Pékin (Chine) en 2008 témoignent d'un engagement en faveur de la MT et des MCA. L'OMS encourage ses 193 états membres à entreprendre, dans la mesure de leurs possibilités, des démarches afin d'intégrer la MT et les MCA dans les systèmes de santé nationaux. Elle offre dans ce contexte son soutien aux états intéressés. Parmi six bureaux régionaux de l'OMS, trois mènent déjà leur propre programme en faveur de la MT. En revanche, le bureau régional de l'Europe ne conduit aucun programme propre en faveur de la MT et des MCA. Du point de vue de la collaboration intensive recherchée de l'OMS avec l'Union européenne, la question de savoir comment le bureau régional souhaite appliquer en Europe les résolutions prises au niveau global au sujet de la MT et des MCA est abordée. Un soutien du bureau régional à travers la dimension internationale de l'OMS ainsi que par les parties prenantes et les communautés d'intérêts des MCA au niveau régional est soumis à discussion.

\section{Einleitung}

"Forschung und Entwicklung in Traditioneller Medizin ist Teil der weltweiten WHO-Strategie und des Aktionsplans für die öffentliche Gesundheit.» (Dr. Margret Chan, Generaldirektorin der Weltgesundheitsorganisation (WHO), in der Eröffnungsrede des ersten $\mathrm{WHO}$-Kongresses für Traditionelle Medizin in Peking, November 2008)

Wenn in den letzten Jahren in den Medien von der WHO die Rede war, so bezog sich dies oft auf Meldungen im Zusammenhang mit SARS, Vogelgrippe (H5N1) und Schweinegrippe (H1N1) sowie die jeweils diskutierten
Massnahmen. In ihrer Verfassung aus dem Jahr 1946 wird als Ziel die Verwirklichung des bestmöglichen Niveaus von Gesundheit bei allen Menschen festgelegt («The objective (...) shall be the attainment by all peoples of the highest possible level of health.»). Dass es für die Umsetzung dieses Zieles mehr braucht als bloss die Bekämpfung oben genannter Epidemien, ist auch der WHO bewusst. Entsprechend umfassend ist das Feld der initiierten Gesundheitsthemen und Projekte. Den an der Ganzheits- und Komplementärmedizin interessierten Mitmenschen stellt sich daher die Frage, was die WHO denn zu diesem Themengebiet beitragen kann. Dieser Fragestellung, d.h. «Können Traditionelle Medizin sowie Komplementäre und Alternative Medizin durch die WHO konkret unterstützt werden?», wird in diesem Essay nachgegangen. Dabei wird zudem auf die WHO-Definitionen zur Traditionellen Medizin (TM) und Komplementären und Alternativen Medizin (CAM) in Kasten 1 hingewiesen.

\section{Kasten 1. Definition der WHO von TM und CAM [11]}

Traditionelle Medizin

TM ist die Gesamtsumme des Wissens, der Fähigkeiten und der Praxis, die auf den Theorien, den Überzeugungen und den Erfahrungen verschiedener indigener Kulturen basieren, und die, ob erklärbar oder nicht, in der Erhaltung der Gesundheit sowie in der Prävention, Diagnose und der Verbesserung oder der Behandlung körperlicher und psychischer Erkrankung verwendet werden.

(Englisches Original: Traditional medicine is the sum total of the knowledge, skills, and practices based on the theories, beliefs, and experiences indigenous to different cultures, whether explicable or not, used in the maintenance of health as well as in the prevention, diagnosis, improvement or treatment of physical and mental illness.)

\section{Komplementäre und Alternative Medizin}

Die Begriffe «Komplementärmedizin» oder «Alternativmedizin» werden in einigen Ländern synonym mit dem Begriff «Traditionelle Medizin» verwendet. Sie beziehen sich auf eine breite Palette von Gesundheitspraktiken, die nicht Teil der Tradition des Landes sind und die nicht in das vorherrschende Gesundheitssystem des Landes integriert sind.

(Englisches Original: The terms 'complementary medicine' or 'alternative medicine' are used interchangeably with 'traditional medicine' in some countries. They refer to a broad set of health care practices that are not part of that country's own tradition and are not integrated into the dominant health care system.) 
In der Europäischen Union (EU) haben 20\% der Bevölkerung eine klare Vorliebe für die komplementärmedizinische Gesundheitsversorgung und weitere 20\% nutzen regelmässig Dienstleistungen der Komplementärmedizin. Über 100 Millionen Bürger in der EU nutzen CAM [1]. In den Vereinigten Staaten von Amerika - mit einer Bevölkerung von ca. 310 Millionen Einwohnern - wurden bei einer nationalen Befragung der Bevölkerung (Stichprobe: 23393 Erwachsene, 9417 Kinder) über die Verwendung von CAM folgende Daten ermittelt: 38,3\% der Erwachsenen und $11,8 \%$ der Kinder verwenden CAM [2]. In einigen asiatischen und afrikanischen Ländern sind $80 \%$ der Bevölkerung von TM als primäre Gesundheitsversorgung abhängig. In vielen sogenannten entwickelten Ländern haben bereits 70-80\% der Bevölkerung verschiedene Formen von CAM in Anspruch genommen. Um angemessen auf diese Ausgangslage eingehen zu können, benennt auch die WHO in einem Factsheet zu TM/CAM diverse Herausforderungen: Dies beginnt bei der uneinheitlichen Terminologie und dem Fehlen eines internationalen Standards. Zudem haben die meisten Länder die TM/CAM noch nicht in die nationale Gesundheitspolitik integriert. Entsprechend ist die Reglementierung und Qualitätssicherung von Produkten und Methoden sowie der Praktizierenden von TM/CAM in vielen Staaten noch verbesserungsbedürftig. Zusätzlich wird das Thema der Nachhaltigkeit angesprochen: Heilpflanzen und weitere Rohstoffe für Heilmittel müssen so gewonnen werden, dass sie trotz Nachfrage über Generationen erhalten bleiben. Dasselbe gelte für den Erhalt der Kenntnisse im Umgang mit den Heilmitteln.

Als Antwort auf diese Ausgangslage und die genannten Herausforderungen propagiert die WHO in diesem Factsheet die Zusammenarbeit mit ihren Mitgliedsstaaten, um die Verwendung der TM zu fördern. Die Ziele sind unter anderem:

- Integration von TM/CAM in die nationalen Gesundheitssysteme, um dadurch Sicherheit und Qualität zu gewährleisten.

- Anerkennung der TM als Teil der primären Gesundheitsversorgung, um den Zugang zur Versorgung zu verbessern und die Fachkenntnisse zu bewahren. Damit die Patientensicherheit gewährleistet werden kann, soll die Fähigkeit und das Wissen der TM/CAM-Praktizierenden verbessert werden [3].

Wie genau diese durchaus ehrbaren und hohen Ziele umgesetzt werden könnten, ob oder wie die WHO konkret Hand bietet bzw. was sie selbst zur Umsetzung beiträgt, wird nachstehend eingehend beleuchtet - im Speziellen, was das Regionalbüro für Europa dazu beitragen könnte.

\section{Welche Aktivitäten unternahm die WHO} im Themenbereich TM/CAM bis heute?

Die WHO mit Sitz in Genf widmet der TM auf ihrer Internetseite ein eigenes Themenfeld (Health Topic) sowie ein eigenes Arbeitsfeld (Areas of Work). Seit 1969 verfasste die WHO insgesamt 15 Resolutionen über TM/CAM, angefangen bei pharmazeutischen Produkten (1969) bis zur Executive-Board-Resolution über TM (2009). Am ersten «WHO-Kongress für Traditionelle Medizin» in Peking (2008) wurde als Abschluss die «Beijing-Deklaration» verfasst, die auf der Internetseite der WHO als «Meilenstein» betitelt wird. Darin beruft sich die WHO auf vorgängige Resolutionen bezüglich TM und kommt zu folgenden sechs Forderungen, die hier aus dem Englischen übersetzt und gekürzt wiedergegeben werden (für den englischen Originaltext siehe Kasten 2):

1. Die Kenntnisse von TM sollten in jedem Land respektiert, erhalten und gefördert werden.

2. Regierungen sollten nationale Verfahrensweisen (Politik: inhaltliche Dimension) und Regulierungen zu TM machen, als Teil des staatlichen Gesundheitssystems und zur Gewährleistung der Anerkennung, Sicherheit und Effektivität von TM.

3. Regierungen, die noch nicht begonnen haben, TM in die nationalen Gesundheitssysteme aufzunehmen, werden aufgerufen, dies zu tun.

4. TM sollte weiterentwickelt werden, gestützt auf Forschung und Innovation im Sinne der entsprechenden globalen Strategie der WHO (WHA61.21, 2008).

5. Regierungen sollten ein System zur Qualifizierung und Anerkennung von TM-Praktizierenden erstellen. Die Praktizierenden sollten ihr Wissen und ihre Fähigkeiten entsprechend den nationalen Bedürfnissen aktualisieren.

6. Die Kommunikation zwischen modernen Medizinern (Conventional Medicine) und TM-Praktizierenden sollte verstärkt werden.

Die 193 Mitgliedsstaaten der WHO werden aufgefordert, zusammen mit weiteren Stakeholdern diese sechs Forderungen betreffend TM/CAM der Beijing-Deklaration umzusetzen und in die nationalen Gesundheitssysteme zu integrieren [4].

Ein Jahr nach dem Kongress in Peking wurde anlässlich der 62. Weltgesundheitsversammlung (World Health Assembly, WHA) der WHO eine Resolution zur TM verfasst. Dabei werden die Mitgliedsstaaten zu Massnahmen aufgerufen (Original: «urges»), die Beijing-Deklaration wenn möglich und unter den jeweiligen landesspezifischen Umständen anzunehmen und einzuführen. Die Forderungen der Beijing-Deklaration werden dabei als Resolution sinngemäss wiederholt. Weiter wird die Generaldirektion ersucht, auf Anfrage der Mitgliedsstaaten die Umsetzung der Deklaration zu unterstützen. Auch solle die WHO- 
Kasten 2. Beijing-Deklaration im englischen Originaltext [4]

I. The knowledge of traditional medicine, treatments and practices should be respected, preserved, promoted and communicated widely and appropriately based on the circumstances in each country.

II. Governments have a responsibility for the health of their people and should formulate national policies, regulations and standards, as part of comprehensive national health systems to ensure appropriate, safe and effective use of traditional medicine.

III. Recognizing the progress of many governments to date in integrating traditional medicine into their national health systems, we call on those who have not yet done so to take action.

IV. Traditional medicine should be further developed based on research and innovation in line with the 'Global strategy and plan of action on public health, innovation and intellectual property' adopted at the Sixty-first World Health Assembly in resolution WHA61.21 in 2008. Governments, international organizations and other stakeholders should collaborate in implementing the global strategy and plan of action.

V. Governments should establish systems for the qualification, accreditation or licensing of traditional medicine practitioners. Traditional medicine practitioners should upgrade their knowledge and skills based on national requirements.

VI. The communication between conventional and traditional medicine providers should be strengthened and appropriate training programmes be established for health professionals, medical students and relevant researchers.

Strategie für TM 2002-2005 aktualisiert und weitergeführt werden. Und abschliessend soll die Kooperation mit bestimmten Forschungsinstituten (WHO Collaboration Centers) und Nichtregierungsorganisationen (engl. «nongovernmental organisation, NGO») verstärkt werden, um Forschung und Ausbildung in TM zu unterstützen. Als Beispiel sei hier das Nationale Zentrum für Komplementäre und Alternative Medizin (NCCAM) in den USA erwähnt [5].

Für die an der Umsetzung der Resolutionen zu TM/ CAM (2003: WHA56.31; 2009: WHA62.13) interessierten Mitgliedsstaaten stellt die WHO diverse Dokumente zur Verfügung: Dabei handelt es sich um Massstäbe (engl. «benchmarks») zu Ausbildung und Anerkennung verschiedener TM/CAM-Berufe. Namentlich finden sich diese Benchmarks für Ayurveda, Naturopathie, Nuad Thai, Osteopathie, TCM, Tuina und Unani-Medizin. Es werden Angaben zum Inhalt der Ausbildung und zum vorgeschlagenen Stundenumfang von Theorie und Praktikum gemacht. Ebenso wird auf die unterschiedliche Vorbildung der Auszubildenden eingegangen [6].

An Grundlagenpapieren und guten Vorsätzen für die TM mangelt es nicht. Auch scheint es, als könne man sich bei Bedarf bei der WHO Unterstützung betreffend TM/ CAM holen - sei es als Land oder als Stakeholder. Die Resolutionen sind jedoch nicht verbindlich, und die WHO verfügt über keine wirklichen Sanktionsmassnahmen bei der Nichtbefolgung durch ihre Mitgliedsstaaten. Es liegt am Souverän des jeweiligen Landes, ob man sich nach dem «Hol-Prinzip» bei den Angeboten der WHO zu TM/CAM bedienen will oder nicht.

\section{Umsetzung der WHO-Strategie bezüglich TM/CAM auf der Ebene der Regionalbüros}

Drei von sechs WHO-Regionen führen ein eigenes Programm für TM/CAM: Dies sind die Region Südostasien, die Region Östliches Mittelmeer (EMRO) und die Region Westlicher Pazifik (WPRO). Die Regionen Europa, Amerika und Afrika führen keine direkten Programme.

In der Region WPRO fand 2009 eine Konferenz des Verbandes Südostasiatischer Nationen (ASEAN) zum Thema TM statt. Dabei entstand die «Erklärung von Bangkok über Traditionelle Medizin in ASEAN» (Bangkok Declaration on Traditional Medicine in ASEAN), in der sich die Teilnehmenden für eine länderübergreifende Zusammenarbeit zugunsten der TM/CAM aussprachen. Dies geschah mit dem Ziel, die Sicherheit, Effektivität und Qualität dieser Fachrichtungen zu gewährleisten und TM in die nationalen Gesundheitssysteme zu integrieren. Auch die Absicht, mit weiteren Partnern zusammenzuarbeiten, um Wissen und Information bezüglich TM auszutauschen, wird deklariert [7].

Die geografisch am nächsten zu Europa gelegene WHORegion, die ein eigenes, seit 1983 laufendes Programm zu TM/CAM hat, ist die Region EMRO. Im Jahr 2002 fand im iranischen Teheran ein Workshop zur Entwicklung regionaler Richtlinien zur Regulierung pflanzlicher Arzneimittel statt. In den Folgejahren entwickelte sich diese Arbeit der Qualitätssicherung weiter, und im November 2005 fand ein Treffen zur Entwicklung von Richtlinien für nationale Gesundheitspolitik in TM/CAM für die EMRORegion statt [8].

Doch wie sieht es in der Region Europa aus? Ein weltweiter Überblick der WHO über die nationale TM/ 
CAM-Gesundheitspolitik kam für die Region Europa zu folgendem Schluss: Von 51 angefragten Staaten haben von den 39 antwortenden Staaten 18\% eine nationale Gesundheitspolitik zu TM/CAM. Dieser Prozentsatz liegt klar unter dem weltweiten Durchschnitt von 32\% gemäss den Angaben der WHO [9]. Auf der Ebene von NGOs oder auch weiteren europäischen Projekten dürfen betreffend CAM einige Aktivitäten beobachtet werden. Als Beispiel: Association of Natural Medicine in Europe (ANME; www.anme.info), das CAMbrellaNetzwerk (www.cambrella.eu), die Camdoc-Vereinigung (www.camdoc.eu) und das European Information Center for Complementary and Alternative Medicine (EICCAM; www.eiccam.eu).

Auf der Internetpräsenz des Regionalbüros der WHO in Europa (WHO/EURO) sind keine spezifischen Aktivitäten betreffend TM/CAM dokumentiert. Auf die direkte Anfrage per E-Mail gab Herr Kees de Joncheere, Regional Adviser Health Technology and Pharmaceuticals des Regionalbüros WHO/EURO, folgende Angaben: Die Frage, weshalb das Regionalbüro kein eigenes Programm für TM/CAM hat, wird begründet mit fehlenden Ressourcen, konkret mit dem Mangel an Personal und Geld. Falls die europäischen Mitgliedsstaaten der WHO die Prioritäten erneut diskutieren und das Führen eines Programms für TM/CAM als vorrangig betrachten sowie entsprechend Geld zur Verfügung stellen, so können Aktivitäten zu TM/ CAM gestartet werden. Auf die Frage nach eventuellem Widerstand innerhalb der WHO/EURO gegen ein solches Programm wird folgende Antwort gegeben: Die WHAResolutionen betreffend TM/CAM gelten auch für die WHO-Region Europa, aber noch seien keine spezifischen regionalen Gesetze verabschiedet worden, und diese Frage wurde bisher auch nicht speziell diskutiert. Ohne ein ausdrückliches Mandat und entsprechende Gelder der Mitgliedsstaaten könnten keine weiterführenden Aktivitäten bezüglich TM/CAM unternommen werden. Die Agenda der WHO werde durch die Mitgliedsstaaten und in Zusammenarbeit mit diesen bestimmt. Weiter gibt de Joncheere an, einer der Gründe, weshalb die Diskussion zu TM/CAM in der Region Europa nicht aufgenommen worden sei, sei das Argument der Kritiker des fehlenden Wirksamkeitsnachweises von TM/CAM. Als Antwort auf die Frage, wie die WHO in Europa unter den aktuellen Umständen die TM/CAM unterstützen könne, macht de Joncheere folgende Angaben: Die WHO nehme im Rahmen ihrer Möglichkeiten eine Interessenvertretung (Advocacy Work) für TM/CAM wahr. Konkret geschehe dies in der Teilnahme an globalen Umfragen zu TM und dadurch, dass einige europäische Länder in der International Regulatory Cooperation for Herbal Medicines (IRCH) vertreten seien.

So weit die Angaben aus dem Regionalbüro der WHO für Europa. Ergänzt werden können diese Angaben noch mit dem Verweis auf die zwei WHO-Kooperationszentren (Collaborating Center, CC) in Europa im Bereich TM/ CAM: das Centre of Research in Bioclimatology, Biotechnologies and Natural Medicine, State University of Milan, Italien, und das National Research Center in Complementary and Alternative Medicine (NAFKAM), University of Tromsø, Norwegen.

Auch wenn das WHO-Regionalbüro in Europa noch kein eigenes Programm für TM/CAM führt, kann es sich mit Blick auf die solide Grundlage, welche die WHO betreffend TM weltweit am Entwickeln ist, lohnen, das Ziel eines regionalen TM/CAM-Programms voranzutreiben. Dies ist zudem unter dem Gesichtspunkt zu betrachten, dass die WHO und die EU ihre Zusammenarbeit verstärken wollen. So sind jährliche Sitzungen hochrangiger Vertreter der EU mit Regionaldirektoren und den beigeordneten Generaldirektoren der WHO vorgesehen. Vorrangige Bereiche sind unter anderem Innovation und Forschung, Gesundheitssicherheit und Gesundheitssysteme [10]. Dass die WHO in diesem Rahmen die Umsetzung ihres weltweiten Programms betreffend TM/ CAM thematisieren könnte, wäre ein zu prüfender Ansatz. Dies könnte zusätzlich durch regionale Stakeholder unterstützt und forciert werden.

\section{Schlussfolgerung}

Fassen wir die Erkenntnisse aus dem Hauptteil in Bezug auf unsere Fragestellung zusammen: Auf globaler Ebene findet sich in Form von Resolutionen und Deklarationen eine in der WHO gut verankerte Unterstützung für die TM/CAM. Ihr wiederholter Appell der Integration von TM/CAM in nationale Gesundheitssysteme kann für Interessierte ein hilfreiches Argumentarium sein. Erfreulich ist das Führen eines Programms für TM/CAM in drei Regionen der WHO.

Mit Blick auf die verschiedenen Regionen der Welt bestehen viele Bestrebungen zur Förderung von TM/CAM, und zwar in östlichen und asiatischen Regionen auch auf Ebene der WHO und der Gesundheitssysteme sowie in der primären Gesundheitsversorgung. In westlichen Regionen erfolgt dies unter anderem auf Forschungsebene und in Interessenverbänden, nicht aber auf der Ebene der WHO-Regionalbüros.

Was also aus westlicher Sicht noch erstrebenswert sein könnte, ist das Führen eines eigenen Programms für TM/ CAM sowohl in der Region Europa als auch in der Region Amerika. Es wäre erfreulich, wenn das Regionalbüro für Europa durch die Mitgliedsstaaten angeregt würde, die globalen Ziele der WHO betreffend TM/CAM in ihrem Wirkungsbereich umzusetzen. Dazu braucht die WHO/ EURO ein Mandat und entsprechende Gelder ihrer Mit- 
glieder. Unterstützt werden kann dies sowohl abwärtsstrukturierend (engl. top-down) durch die globale Ebene der WHO als auch aufwärtsstrukturierend (engl. bottomup) durch regionale Stakeholder - dies besonders auch unter dem Aspekt der geplanten verstärkten Zusammenarbeit von EU und WHO. Hierfür ist die koordinierte Zusammenarbeit gewünscht: länderübergreifend, interdisziplinär, zwischen den einzelnen Regionen und auch mit allen beteiligten Stakeholdern - inklusive der politischen Organe, dem gezielten Lobbying, der Kooperation von akademischen und nichtakademischen Heilberufen und dem Einbezug der Konsumenten/Patienten. Ein breit abgestützter, koordinierter und von allen Interessengrup- pen getragener paneuropäischer TM/CAM-Dachverband könnte dabei hilfreich sein.

Die Bestärkung und Ermutigung der WHO durch TM/ CAM-Interessenverbände, den auf globaler Ebene eingeschlagenen Weg betreffend TM weiter zu beschreiten, sei hier ausdrücklich empfohlen, damit diese Strategie weltweit erfolgreich umgesetzt werden kann.

\section{Disclosure Statement}

Es bestehen keine Interessenkonflikte.

\section{Literatur}

1 EICCAM Portal: European Information Centre for Complementary und Alternative Medicine. 2009. www.eiccam.eu/portal.html.

2 NCCAM: National Center for Complementary and Alternative Medicine. The Use of Complementary and Alternative Medicine in the United States. 2007, Update 2008. www.nccam.nih. gov/news/camstats/2007/camsurvey_fs1.htm.

3 Factsheet WHO $\mathrm{N}^{\circ} 134$ : Traditional Medicine. 2008.www.who.int/mediacentre/factsheets/fs134/ en/index.html.

4 Beijing Declaration. 2008. www.who.int/ medicines/areas/traditional/congress/beijing_ declaration/en/index.html.
5 WHA62.13 Resolution: Sixty-second World Health Assembly: Agenda item 12.4: Traditional Medicine. 2009. apps.who.int/gb/ebwhal pdf_files/A62/A62_R13-en.pdf.

6 Benchmarks for Training in Traditional/ Complementary and Alternative Medicine. 2010. www.who.int/medicines/areas/traditional/ trm_benchmarks/en/index.html.

7 Bangkok Declaration on Traditional Medicine in ASEAN. 2009. www.aseansec.org/Doc-Bangkok-Declaration-on-Traditional-Medicine.pdf.

8 EMRO.WHO: Programme areas: Traditional Medicines: Regional work in the area of Traditional Medicines. 2011. www.emro.who.int/ edb/medicines_traditional_emr.htm.
9 WHO Report: National Policy on Traditional Medicine and Regulation of Herbal Medicines: Global Survey. 2005. apps.who.int/ medicinedocs/pdf/s7916e/s7916e.pdf.

10 EURO.WHO: Über die Regionaldirektorin: Weichenstellung für die Zusammenarbeit zwischen WHO und EU. 2011. www.euro. who.int/de/who-we-are/regional-director/news/ news/2012/01/setting-the-agenda-for-who-andeuropean-union-collaboration.

11 WHO: Medicines. Traditional Medicine: Definitions. 2011. www.who.int/medicines/areas/ traditional/definitions/en/index.html. 